\title{
RACSAM
}

Rev. R. Acad. Cien. Serie A. Mat.

VOL. 202 (2), 2008, pp. 335-353

Matemática Aplicada / Applied Mathematics

\section{Actions on Environment under uncertainty: stochastic formulation and the associated deterministic problem}

\author{
J. I. Díaz and Ch. Faghloumi
}

\begin{abstract}
An application of the results of this paper proves that there is not always an economic benefit when destroying the environment for planting an alternative industrial project. Our criterion, to act, to delay or to deny the industrial investment over the environment, is given in terms of the free boundary associated to a deterministic degenerate obstacle problem (on an unbounded domain) associated to the stochastic optimal control problem formulated, initially, in terms of some suitable stochastic diffusion processes. The localizing estimates on the free boundary are obtained through a suitable spatial change of variables and by working with a suitable distance associated to the coefficient of the elliptic operator.

\section{Acciones sobre el Medio Ambiente bajo incertidumbre: formulación estocástica y el problema determinista asociado}

Resumen. Una interpretación de los resultados de este trabajo muestra que no siempre hay beneficio económico cuando se destruye el medio ambiente para la implantación de un proyecto industrial alternativo. Nuestro criterio, de actuar, retrasar o negar la inversión industrial sobre el medio ambiente, viene dado en términos de la frontera libre asociada a un problema de obstáculo determinista degenerado (sobre un dominio no acotado) asociado al problema estocástico de control óptimo formulado, inicialmente, en términos de ciertos procesos de difusión estocásticos.
\end{abstract}

\section{Introduction}

The aim of this study is to prove that there is not always an economic benefit when destroying the environment for planting an industrial project alternative to the environment. We give a criterion, in terms of a free boundary, to act and to delay or to deny the industrial investment over the environment. To model the problem, let's consider the environment and an alternative project as two economical goods for which the instant benefits, to time $t$, are given by $\mathcal{X}(t)$ and $\mathcal{Y}(t)$ respectively. This paper improves the results of [12] in several aspects (more general data, more precise estimates, etc.).

We assume that the two benefits, associated to the environment and to the alternative project, are both, in general, not constant. Suppose that the benefits of the environment are completely destroyed once the alternative project comes in action. We assume that the benefits $\mathcal{X}(t)$ and $\mathcal{Y}(t)$ obey to certain diffusion processes of the type

$$
\begin{cases}\mathrm{d} \mathcal{X}(t)=\mu_{1} \mathcal{X}(t) \mathrm{d} t+\sqrt{2} \sigma_{1} \mathcal{X}(t) \mathrm{d} \mathcal{B}_{1}(t), & \mathcal{X}(0)=x \in \mathbb{R} \\ \mathrm{d} \mathcal{Y}(t)=\mu_{2} \mathcal{Y}(t) \mathrm{d} t+\sqrt{2} \sigma_{2} \mathcal{Y}(t) \mathrm{d} \mathcal{B}_{2}(t), & \mathcal{Y}(0)=y \in \mathbb{R}\end{cases}
$$

Presentado por / Submitted by Manuel López Pellicer.

Recibido / Received: 13 de noviembre de 2007. Aceptado / Accepted: 15 de octubre de 2008.

Palabras clave / Keywords: degenerate obstacle problem, unbounded domain, stochastic optimal control problem, environmental economy

Mathematics Subject Classifications: 35T35,60H15,93E20,91B76

(C) 2008 Real Academia de Ciencias, España. 
in a probability space $(\mathcal{O}, \mathcal{F}, \mathbb{P})$, where $\left\{\mathcal{B}_{i}(t)\right\}_{t \geq 0}$ are standard one-dimensional Brownian movements and $\left\{\mathcal{F}_{t}\right\}_{t \geq 0} \subset \mathcal{F}$ is the associated filtration adapted to $\left\{\mathcal{B}_{i}(t)\right\}_{t \geq 0}, i=1,2$. In fact, the initial model is governed by

$$
\begin{cases}\mathrm{d} \mathcal{X}(t)=\mu_{1} \mathcal{X}(t) \mathrm{d} t+\widehat{\sigma} \mathcal{X}(t)(\mathrm{d} \mathcal{W}(t)+\widehat{\varrho} \mathrm{d} \mathcal{Z}(t)), & \mathcal{X}(0)=x \in \mathbb{R}, \\ \mathrm{d} \mathcal{Y}(t)=\mu_{2} \mathcal{Y}(t) \mathrm{d} t+\widehat{\sigma}_{2} \mathcal{Y}(t)(\widehat{\varrho} \mathrm{d} \mathcal{W}(t)+\mathrm{d} \mathcal{Z}(t)), & \mathcal{Y}(0)=y \in \mathbb{R}\end{cases}
$$

where $\{\mathcal{W}(t)\}_{t \geq 0}$ and $\{\mathcal{Z}(t)\}_{t \geq 0}$ are two standard Brownian movements with covariant matrix

$$
\Sigma_{\mathcal{W}, \mathcal{Z}}(t)=2 t\left(\begin{array}{cc}
1 & \widehat{\varrho} \\
\widehat{\varrho} & 1
\end{array}\right)
$$

i.e. $\mathcal{W}(t)$ and $\mathcal{Z}(t)$ have a constant correlation coefficient $\widehat{\varrho} \in]-1,1\left[\right.$. Then the $\left\{\mathcal{B}_{i}(t)\right\}_{t \geq 0}, i=1,2$ introduced by

$$
\left\{\begin{array}{l}
\mathcal{B}_{1}(t)=k(\mathcal{W}(t)+\widehat{\varrho} \mathcal{Z}(t)), \\
\mathcal{B}_{2}(t)=k(\widehat{\varrho} \mathcal{W}(t)+\mathcal{Z}(t)),
\end{array}\right.
$$

have the following correlation matrix

$$
\Sigma_{\mathcal{B}_{1}, \mathcal{B}_{2}}(t)=2 t k^{2}\left(\begin{array}{ll}
1+3 \widehat{\varrho}^{2} & \widehat{\varrho}\left(3+\widehat{\varrho}^{2}\right) \\
\widehat{\varrho}\left(3+\widehat{\varrho}^{2}\right) & 1+3 \widehat{\varrho}^{2}
\end{array}\right) .
$$

Then, the new Brownian movements have a correlation coefficient $\varrho$, independent of $k$ and $t$, given by

$$
\varrho \doteq \widehat{\varrho}\left(\frac{3+\widehat{\varrho}^{2}}{1+3 \widehat{\varrho}^{2}}\right)
$$

and a better determination coefficient thanks to

$$
\varrho=\widehat{\varrho}^{2}\left(\frac{3+\widehat{\varrho}^{2}}{1+3 \widehat{\varrho}^{2}}\right)^{2}>\widehat{\varrho}^{2} .
$$

Finally, we take $k$ as to work with standard Brownian movements and $\left\{\mathcal{B}_{i}(t)\right\}_{t \geq 0}$ result of the following form

$$
\left\{\begin{array}{l}
\mathcal{B}_{1}(t)=\frac{1}{\sqrt{1+3 \widehat{\varrho}^{2}}}(\mathcal{W}(t)+\widehat{\varrho} \mathcal{Z}(t)), \\
\mathcal{B}_{2}(t)=\frac{1}{\sqrt{1+3 \widehat{\varrho}^{2}}}(\widehat{\varrho} \mathcal{W}(t)+\mathcal{Z}(t)) .
\end{array}\right.
$$

Then, we can write the problem in the form (1) with

$$
\sigma_{i}=\frac{\widehat{\sigma}_{i} \sqrt{1+3 \varrho^{2}}}{\sqrt{2}}, \quad i=1,2 .
$$

We consider now the conditions given by the utility function $J$ defined by

$$
J(x, y ; T) \doteq \mathbb{E}\left[\int_{0}^{+\infty} \mathrm{e}^{-\alpha s}\left(\mathbb{I}_{] 0, T[}(s) f(\mathcal{X}(s))+\mathbb{1}_{] T, \infty[}(s) \mathcal{Y}(s)\right) \mathrm{d} s\right],
$$

where $f$ and $\alpha$ are a function and a constant, respectively. Our main goal is to study the optimal function $v$ given as the maximum of the utility function among the different time horizons $\mathrm{T}$

$$
v(x, y) \doteq \max _{T}\{J(x, y, T)\}
$$

Here, the pair $(x, y)$ runs the open unbounded domain

$$
\Omega=\left\{(x, y) \in \mathbb{R}^{2}: x>0, y>0\right\} .
$$


It is easy to show that the optimal function $v$ satisfy the obstacle problem

$$
\min \{-\mathcal{L} v+\alpha v-f(x), v-h\}=0 \quad \text { in } \Omega,
$$

where $\mathcal{L}$ is the differential operator associated to the multidiffusion $\left(X_{t}, Y_{t}\right)$ given by

$$
\mathcal{L} v \doteq \sigma_{1}^{2} x^{2} v_{x x}+\sigma_{2}^{2} y^{2} v_{y y}+2 \sigma_{1} \sigma_{2} \varrho x y v_{x y}+\mu_{1} x v_{x}+\mu_{2} y v_{y}
$$

and

$$
h(x, y) \doteq E\left[\int_{0}^{\infty} \mathcal{Y}(s) e^{-\alpha s} \mathrm{~d} s\right]=\frac{y}{(\lambda-1)\left(\sigma_{2}^{2} \lambda+\mu_{2}\right)}
$$

with

$$
\lambda \doteq \frac{1}{2}\left(\left(1-\frac{\mu_{2}}{\sigma_{2}}\right)-\sqrt{\left(1-\frac{\mu_{2}}{\sigma_{2}}\right)^{2}+\frac{4 \alpha}{\sigma^{2}}}\right) .
$$

Indeed,

$$
\begin{aligned}
v(x, y) & \geq \mathcal{J}(x, y ; \mathrm{T}+k) \\
& \geq \mathbb{E}\left[\int_{0}^{k} \mathrm{e}^{-\alpha s} f(\mathcal{X}(s)) \mathrm{d} s+\int_{k}^{\mathrm{T}+k} \mathrm{e}^{-\alpha s} f(\mathcal{X}(s)) \mathrm{d} s+\int_{\mathrm{T}+k}^{+\infty} \mathcal{Y}(s) \mathrm{e}^{-\alpha s} \mathrm{~d} s\right] \\
& \geq \mathbb{E}\left[\int_{0}^{k} \mathrm{e}^{-\alpha s} f(\mathcal{X}(s)) \mathrm{d} s\right]+\mathrm{e}^{-\alpha k}\left(\mathbb{E}\left[\int_{0}^{\mathrm{T}} \mathrm{e}^{-\alpha \hat{s}} f(\mathcal{X}(\hat{s}+k)) \mathrm{d} \hat{s}+\int_{\mathrm{T}}^{+\infty} \mathcal{Y}(\hat{s}+k) \mathrm{e}^{-\alpha s} \mathrm{~d} \hat{s}\right]\right) \\
& \geq \mathbb{E}\left[\int_{0}^{k} e^{-\alpha s} f(\mathcal{X}(s)) \mathrm{d} s\right]+\mathrm{e}^{-\alpha k} \mathcal{J}(\mathcal{X}(k), \mathcal{Y}(k) ; \mathrm{T}), \quad \mathrm{T} \in \mathbb{R}_{+},
\end{aligned}
$$

i.e.

$$
\frac{v(x, y)-\mathrm{e}^{-\alpha k} \mathbb{E}[v(\mathcal{X}(k), \mathcal{Y}(k))]}{k} \geq \frac{1}{k} \mathbb{E}\left[\int_{0}^{k} \mathrm{e}^{-\alpha s} f(\mathcal{X}(s)) \mathrm{d} s\right], \quad(x, y) \in \mathbb{R}^{2} .
$$

On the other hand, assume that $v \geq h$, then, for any $\epsilon>0$ there exists $\mathrm{T}_{0} \geq 0$ such that $\mathrm{T}^{\epsilon}=\mathrm{T}_{0}+k$ verifies the inequality

$$
v(x, y) \leq \mathcal{J}(x, y ; \mathrm{T} \epsilon)+\epsilon .
$$

Then

$$
\begin{aligned}
& v(x, y) \leq \mathcal{J}\left(x, y ; \mathrm{T}_{0}+k\right)+\epsilon \\
& \leq \mathbb{E}\left[\int_{0}^{k} \mathrm{e}^{-\alpha s} f(\mathcal{X}(s)) \mathrm{d} s+\int_{k}^{\mathrm{T}_{0}+k} \mathrm{e}^{-\alpha s} f(\mathcal{X}(s)) \mathrm{d} s+\int_{\mathrm{T}_{0}+h}^{+\infty} \mathcal{Y}(s) \mathrm{e}^{-\alpha s} \mathrm{~d} s\right]+\epsilon \\
& \leq \mathbb{E}\left[\int_{0}^{k} \mathrm{e}^{-\alpha s} f(\mathcal{X}(s)) \mathrm{d} s\right] \\
& \\
&+\mathrm{e}^{-\alpha k}\left(\mathbb{E}\left[\int_{0}^{\mathrm{T}_{0}} \mathrm{e}^{-\alpha \hat{s}} f(\mathcal{X}(\hat{s}+k)) \mathrm{d} \hat{s}+\int_{\mathrm{T}_{0}}^{+\infty} \mathcal{Y}(\hat{s}+k) \mathrm{e}^{-\alpha s} \mathrm{~d} \hat{s}\right]\right)+\epsilon \\
& \leq \mathbb{E}\left[\int_{0}^{k} \mathrm{e}^{-\alpha s} f(\mathcal{X}(s)) \mathrm{d} s\right]+\mathrm{e}^{-\alpha k} \mathcal{J}\left(\mathcal{X}(k), \mathcal{Y}(k) ; \mathrm{T}_{0}\right)+\epsilon \\
& \leq \mathbb{E}\left[\int_{0}^{k} \mathrm{e}^{-\alpha s} f(\mathcal{X}(s)) \mathrm{d} s\right]+\mathrm{e}^{-\alpha k} \mathbb{E}[v(\mathcal{X}(k), \mathcal{Y}(k))]+\epsilon .
\end{aligned}
$$


Passing to the limit in $\epsilon$

$$
v(x, y) \leq \mathbb{E}\left[\int_{0}^{k} \mathrm{e}^{-\alpha s} f(\mathcal{X}(s)) \mathrm{d} s\right]+\mathrm{e}^{-\alpha k} \mathbb{E}[v(\mathcal{X}(k), \mathcal{Y}(k))]
$$

i.e.

$$
\frac{v(x, y)-\mathrm{e}^{-\alpha k} \mathbb{E}[v(\mathcal{X}(k), \mathcal{Y}(k))]}{k} \leq \frac{1}{k} \mathbb{E}\left[\int_{0}^{k} \mathrm{e}^{-\alpha s} f(\mathcal{X}(s)) \mathrm{d} s\right], \quad(x, y) \in \mathbb{R}^{2} .
$$

Now, by classical arguments we can show that $v$ is solution of the problem (4). One of the main objectives of the next section is the study of the existence and qualitative properties of the function $v: \Omega \rightarrow \mathbb{R}$ through the consideration of the elliptic obstacle problem (4). In fact, like in the illustrative example, this problem gives rise to free boundary which is the boundary of the coincidence set (the set of the points $(x, y) \in \Omega$ where $v$ coincides with the obstacle $h$ ). Sometimes this free boundary corresponds to the graph of a function $g:[0, \infty) \rightarrow \mathbb{R}^{+}$, leaving to the, so called, strong formulation

$$
\begin{cases}-\mathcal{L} v+\alpha v \geq f(x) \quad \text { and } \quad v(x, y)=h(y) \quad \text { if } x \geq g(y) \\ -\mathcal{L} v+\alpha v=f(x) \quad \text { and } \quad v(x, y) \geq h(y) \quad \text { if } x \leq g(y)\end{cases}
$$

(Note that $g$ is an a priori unknown function). Another formulation of the nonlinear obstacle problem (4) is now available in terms of multivalued operators. It is known (see e.g. [5] as a general reference) that the obstacle problem can be written as

$$
-\mathcal{L} v+\alpha v+\gamma(v-h) \ni f(x) \quad \text { in } \Omega,
$$

where $\gamma$ is the maximal monotone graph of $\mathbb{R}^{2}$ given by

$$
\gamma(u)= \begin{cases}\{0\} & \text { if } u>0 \\ (-\infty, 0] & \text { if } u=0 \\ \emptyset & \text { if } u<0\end{cases}
$$

with $\emptyset$ the empty set. Since the operator $\mathcal{L}(v)$ is not, a priori, in divergence form, many authors used the notion of viscosity solution, introduced by M. G. Crandall and P. L. Lions (see, e.g. the exposition made in [8]). However, here we can address the problem in different ways motivated by the linearity and the special properties of the operator $\mathcal{L}(v)$. So, one of the more important ingredients of our treatment is that really $\mathcal{L}(v)$ may be written with its main part in divergence form. To do that, we introduce the notations

$$
\mathbf{A}(x, y)=\left(\begin{array}{cc}
\sigma_{1}^{2} x^{2} & \sigma_{1} \sigma_{2} \varrho x y \\
\sigma_{1} \sigma_{2} \varrho x y & \sigma_{2}^{2} y^{2}
\end{array}\right) \text { and } \mathbf{b}(x, y)=\left(\begin{array}{c}
\left(\mu_{1}-2 \sigma_{1}^{2}-\sigma_{1} \sigma_{2} \varrho\right) x \\
\left(\mu_{2}-2 \sigma_{2}^{2}-\sigma_{1} \sigma_{2} \varrho\right) y
\end{array}\right) .
$$

Then, it is easy to see that

$$
\mathcal{L}(v)=\operatorname{div}(\mathbf{A} \nabla v)+\mathbf{b} \cdot \nabla v .
$$

A polemic fact, which appears in this problem (4), as well as in other ones associated to the similar operators to the operator's $\mathcal{L}(v)$ (see, e.g. [4]), is the absence of boundary conditions in the statement. A natural question is either to prescribe or not some boundary values for $v$ or $\nabla v$ on the boundary of $\Omega$. Here we shall adopt the philosophy to assume that the operator $\mathcal{L}(v)$ always prescribes the natural boundary condition

$$
(\mathbf{A} \nabla u) \cdot \nu=0 \quad \text { in } \partial \Omega,
$$

where $\nu$ is the outgoing unit normal vector.

Note that this boundary condition is automatically satisfied thanks to the special form of the matrix $\mathbf{A}(x, y)$. 
Now $h=h(x, y)$ is taken as a general obstacle function. It is useful to introduce the following change of variable $u=v-h$. Then the problem under consideration is given by

$$
(\mathcal{P}) \quad \begin{cases}-\operatorname{div}(\mathbf{A} \nabla u)+\mathbf{b} \cdot \nabla u+\alpha u+\gamma(u) \ni G & \text { in } \Omega, \\ (\mathbf{A} \nabla u) \cdot \nu=0 & \text { on } \partial \Omega,\end{cases}
$$

where $G(x, y)=f(x)-H(x, y)$ and

$$
h(x, y)=E\left[\int_{0}^{\infty} H\left(X_{s}, Y_{s}\right) e^{-\alpha s} d s\right]
$$

i.e. $h$ satisfies

$$
\begin{cases}-\operatorname{div}(\mathbf{A} \nabla h)+\mathbf{b} \cdot \nabla h+\alpha h=H & \text { in } \Omega \\ (\mathbf{A} \nabla h) \cdot \nu=0 & \text { on } \partial \Omega .\end{cases}
$$

In fact, under more generality we shall suppose that $G$ is a given function. Note that as the domain $\Omega$ is not bounded then the natural boundary condition (7) has to be supplemented by some assumption about the growth conditions of the solution $v$, for $(x, y)$ large enough. Usually, this is implicated by the type of growth conditions assumed on the datum $G(x, y)$. In our case, we shall always assume that

$$
\text { there exists } m_{1}>1 \text { and } m_{2}>1 \text { such that } w^{\frac{1}{2}} G \in L^{2}(\Omega) \text {, }
$$

where the weight $w$ is defined by

$$
w(x, y)=\left(1+x^{2}\right)^{-m_{1}}\left(1+y^{2}\right)^{-m_{2}},
$$

in other words

$$
\int_{\Omega}\left(1+x^{2}\right)^{-m_{1}}\left(1+y^{2}\right)^{-m_{2}} G^{2}(x, y) \mathrm{d} x \mathrm{~d} y<\infty .
$$

We point out that when working with in semi-linear problems on unbounded domains, several authors (see, e.g. [7]) adopt different weights $\left(w(x, y)=\mathrm{e}^{-\lambda|(x, y)|^{p}}\right.$, for some $\left.p>0\right)$ motivated by the transformation $(x, y)=\left(\log x^{\prime}, \log y^{\prime}\right)$. But in our case this transformation is meaningless on the boundary of the domain $\Omega$.

\section{Existence and uniqueness of the solution}

Before to introduce the weak formulation of problem $(\mathcal{P})$ we introduce the Hilbert spaces

$$
L_{w}^{2}(\Omega)=\left\{v: w^{\frac{1}{2}} v \in L^{2}(\Omega)\right\}
$$

with the norm

$$
\|u\|_{L_{w}^{2}(\Omega)}=\left\|w^{\frac{1}{2}} u\right\|_{L^{2}(\Omega)}
$$

and

$$
H_{w}^{1}(\Omega, \mathbf{A})=\left\{v: v \in L_{w}^{2}(\Omega), x v_{x} \in L_{w}^{2}(\Omega), y v_{y} \in L_{w}^{2}(\Omega)\right\}
$$

with its norm:

$$
\|u\|_{H_{w}^{1}(\Omega, \mathbf{A})}^{2}=\|u\|_{L_{w}^{2}(\Omega)}^{2}+\left\|x u_{x}\right\|_{L_{w}^{2}(\Omega)}^{2}+\left\|y u_{y}\right\|_{L_{w}^{2}(\Omega)}^{2} .
$$

We define the convex set $\mathcal{K}$

$$
\mathcal{K}=\left\{v \in H_{w}^{1}(\Omega, \mathbf{A}), v \geq 0 \text { on } \Omega\right\}
$$


Then it is easy to see that any regular solution of $(\mathcal{P})$ satisfies also the problem under the weaker formulation defined in the following terms:

$$
(\mathcal{W P})\left\{\begin{array}{l}
u \in \mathcal{K}, \\
a(u, v-u) \geq L(v-u), \quad \text { for any } v \in \mathcal{K},
\end{array}\right.
$$

where

$$
\begin{aligned}
a(u, v)= & \int_{\Omega} w\left\{\nabla v \cdot \mathbf{A} \cdot \nabla u+\left[\left(\frac{1}{w} \nabla w \cdot \mathbf{A}-\mathbf{b}\right) \cdot \nabla u\right] v+\alpha u v\right\} \mathrm{d} x \mathrm{~d} y \\
= & \int_{\Omega} \sigma_{1}^{2} x^{2} w u_{x} v_{x}+\sigma_{2}^{2} y^{2} w u_{y} v_{y}+\sigma_{1} \sigma_{2} \varrho x y w u_{x} v_{y}+\sigma_{1} \sigma_{2} \varrho x y w u_{y} v_{x} \\
& \quad-\int_{\Omega} w\left(\mu_{1}+2 \sigma_{1}^{2}\left(m_{1} \frac{x^{2}}{1+x^{2}}-1\right)+\sigma_{1} \sigma_{2} \varrho\left(2 m_{2} \frac{y^{2}}{1+y^{2}}-1\right)\right) u_{x} v \\
& \quad-\int_{\Omega} w\left(\mu_{2}+2 \sigma_{2}^{2}\left(m_{2} \frac{y^{2}}{1+y^{2}}-1\right)+\sigma_{1} \sigma_{2} \varrho\left(2 m_{1} \frac{x^{2}}{1+x^{2}}-1\right)\right) u_{y} v+\alpha w u v
\end{aligned}
$$

and

$$
L(v)=\int_{\Omega} G v w \mathrm{~d} x \mathrm{~d} y
$$

Reciprocally, it can be shown that any solution of $(\mathcal{W P})$, being twice differentiable, satisfies also $(\mathcal{P})$ for almost every point (see, the exposition given in [6]). Our existence results require some technical conditions on the constants $\mu$ and $m$ appearing in the operator $\mathcal{L}(v)$ and the growth condition on $G$.

Theorem 1 Assume (8) and

$$
\alpha>\alpha_{0}
$$

given by

$$
\begin{aligned}
\alpha_{0}=( & \left.m_{1}+1\right) \frac{\mu_{1}}{4}+\left(m_{2}+1\right) \frac{\mu_{2}}{4}+\frac{\sigma_{1}^{2}}{2}\left(m_{1}^{2}-1\right)+\frac{\sigma_{2}^{2}}{2}\left(m_{2}^{2}-1\right) \\
+ & \frac{\sigma_{1} \sigma_{2}|\varrho|}{4}\left(4 m_{1} m_{2}+5 m_{1}+5 m_{2}-2\right)
\end{aligned}
$$

Then, there exists a unique weak solution $u \in \mathcal{K}$ of problem $(\mathcal{W P})$.

PROOF. As in [12], we began by introducing the change of space variables that transforms the unbounded set $\Omega$ in a bounded. Let $\mathcal{F}$ be such transformation which we assume to be given by

$$
\begin{aligned}
& \mathcal{F}: \quad \Omega \quad \rightarrow\left(0, \frac{\pi}{2}\right) \times\left(0, \frac{\pi}{2}\right) \\
& (x, y) \rightarrow(\theta, \beta)=\mathcal{F}(x, y)=(\arctan x, \arctan y) \text {. }
\end{aligned}
$$

To keep a coherent notation we rename

$$
\mathcal{F}(\Omega)=\left(0, \frac{\pi}{2}\right) \times\left(0, \frac{\pi}{2}\right) .
$$

Note that the assumption (8) is equivalent to

$$
\text { there exists } \quad \widehat{m}_{1}, \widehat{m}_{2}>0 \quad \text { such that } \quad \omega^{\frac{1}{2}} \widehat{G} \in L^{2}(\mathcal{F}(\Omega)),
$$

where $\widehat{m}_{1}=m_{1}-1, \widehat{m}_{2}=m_{2}-1, \omega(\theta, \beta)=\cos ^{2 \widehat{m}_{1}}(\theta) \cos ^{2 \widehat{m}_{2}}(\beta)$ and $\widehat{G}(\theta, \beta)=G\left(\mathcal{F}^{-1}(\theta, \beta)\right)$, i.e.

$$
\int_{\mathcal{F}(\Omega)} \omega(\theta, \beta) \widehat{G}^{2}(\theta, \beta) \mathrm{d} \theta \mathrm{d} \beta<\infty .
$$


We also introduce the notation

$$
\begin{aligned}
& \mathbf{S}=\left(\begin{array}{cc}
\frac{\sigma_{1}^{2}}{4} \sin ^{2} 2 \theta & \frac{\sigma_{1} \sigma_{2} \varrho}{4} \sin 2 \theta \sin 2 \beta \\
\frac{\sigma_{1} \sigma_{2} \varrho}{4} \sin 2 \theta \sin 2 \beta & \frac{\sigma_{2}^{2}}{4} \sin ^{2} 2 \beta
\end{array}\right), \\
& \mathbf{p}=\left(\begin{array}{c}
\frac{\sin 2 \theta}{2}\left(\mu_{1}-2 \sigma_{1}^{2} \cos ^{2} \theta-\sigma_{1} \sigma_{2} \varrho \cos 2 \beta\right) \\
\frac{\sin 2 \beta}{2}\left(\mu_{2}-2 \sigma_{2}^{2} \cos ^{2} \beta-\sigma_{1} \sigma_{2} \varrho \cos 2 \theta\right)
\end{array}\right) .
\end{aligned}
$$

The formulation of the model in the new spatial variables is the following

$$
(\widehat{\mathcal{P}}) \begin{cases}-\operatorname{div}_{(\theta, \beta)}(\mathbf{S} \nabla u)-\mathbf{p} \cdot \nabla u+\alpha u+\gamma(u) \ni \widehat{G} & \text { in } \mathcal{F}(\Omega), \\ (\mathbf{S} \nabla u) \cdot \widetilde{\nu}=0 & \text { on } \partial \mathcal{F}(\Omega),\end{cases}
$$

where now $\widetilde{\nu}$ represents the unit outgoing normal vector in $\partial \mathcal{F}(\Omega)$. The weak formulation of $(\widehat{\mathcal{P}})$ starts, again, by introducing the Hilbert spaces associated to the problem:

$$
L_{\omega}^{2}(\mathcal{F}(\Omega))=\left\{v: \omega^{\frac{1}{2}} v \in L^{2}(\mathcal{F}(\Omega))\right\}
$$

with the norm:

$$
\|u\|_{L_{\omega}^{2}(\mathcal{F}(\Omega))}=\left\|\omega^{\frac{1}{2}} u\right\|_{L^{2}(\mathcal{F}(\Omega))},
$$

and

$$
H_{\omega}^{1}(\mathcal{F}(\Omega), \mathbf{S})=\left\{v: v \in L_{\omega}^{2}(\mathcal{F}(\Omega)),(\sin 2 \theta) v_{\theta} \in L_{\omega}^{2}(\mathcal{F}(\Omega)),(\sin 2 \beta) v_{\beta} \in L_{\omega}^{2}(\mathcal{F}(\Omega))\right\}
$$

of norm

$$
\|u\|_{H_{\omega}^{1}(\mathcal{F}(\Omega), \mathbf{S})}^{2}=\|u\|_{L_{\omega}^{2}(\mathcal{F}(\Omega))}^{2}+\left\|(\sin 2 \theta) v_{\theta}\right\|_{L_{\omega}^{2}(\mathcal{F}(\Omega))}^{2}+\left\|(\sin 2 \beta) v_{\beta}\right\|_{L_{\omega}^{2}(\mathcal{F}(\Omega))}^{2} .
$$

We introduce the notation

$$
\mathcal{F}\left(H_{w}^{1}(\Omega, \mathbf{A})\right)=\left\{\mathcal{F}(v): v \in H_{w}^{1}(\Omega, \mathbf{A})\right\} \text { and } \widehat{\mathcal{K}}=\left\{v \in H_{\omega}^{1}(\mathcal{F}(\Omega), \mathbf{S}), v \geq 0\right\}
$$

It is easy to see that

$$
\mathcal{F}\left(H_{w}^{1}(\Omega, \mathbf{A})\right)=H_{\omega}^{1}(\mathcal{F}(\Omega), \mathbf{S})
$$

and

$$
\mathcal{F}(\mathcal{K})=\widehat{\mathcal{K}}
$$

Then, the weak formulation of $(\widehat{\mathcal{P}})$ can be stated in the following terms

$$
(\mathcal{W} \widehat{\mathcal{P}}) \quad\left\{\begin{array}{l}
u \in \widehat{\mathcal{K}} \\
\widehat{a}(u, v-u) \geq \widehat{L}(u, v-u) \quad \forall v \in \widehat{\mathcal{K}}
\end{array}\right.
$$


where

$$
\begin{aligned}
\widehat{a}(u, v)= & \int_{\mathcal{F}(\Omega)} \omega\left\{\nabla v \cdot \mathbf{S} \cdot \nabla u+\left[\left(\frac{1}{\omega} \nabla \omega \cdot \mathbf{S}-\mathbf{p}\right) \cdot \nabla u\right] v+\alpha u v\right\} \mathrm{d} \theta \mathrm{d} \beta \\
= & \int_{\mathcal{F}(\Omega)} \frac{\sigma_{1}^{2}}{4} \omega \sin ^{2}(2 \theta) u_{\theta} v_{\theta}+\frac{\sigma_{2}^{2}}{4} \omega \sin ^{2}(2 \beta) u_{\beta} v_{\beta} \\
& +\int_{\mathcal{F}(\Omega)} \frac{\sigma_{1} \sigma_{2} \varrho}{4} \sin (2 \theta) \omega \sin (2 \beta) u_{\beta} v_{\theta}+\frac{\sigma_{1} \sigma_{2} \varrho}{4} \omega \sin (2 \theta) \sin (2 \beta) u_{\theta} v_{\beta} \\
& -\int_{\mathcal{F}(\Omega)}\left[\mu_{1}-2 \sigma_{1}^{2}\left(1-\left(\widehat{m}_{1}+1\right) \sin ^{2}(\theta)\right)-\sigma_{1} \sigma_{2} \varrho\left(1-2\left(\widehat{m}_{2}+1\right) \sin ^{2}(\beta)\right)\right] \frac{\sin (2 \theta)}{2} \omega u_{\theta} v \\
& -\int_{\mathcal{F}(\Omega)}\left[\mu_{2}-2 \sigma_{2}^{2}\left(1-\left(\widehat{m}_{2}+1\right) \sin ^{2}(\beta)\right)-\sigma_{1} \sigma_{2} \varrho\left(1-2\left(\widehat{m}_{1}+1\right) \sin ^{2}(\theta)\right)\right] \frac{\sin (2 \beta)}{2} \omega u_{\beta} v \\
& +\int_{\mathcal{F}(\Omega)} \alpha \omega u v
\end{aligned}
$$

and

$$
\widehat{L}(v)=\int_{\mathcal{F}(\Omega)}(\omega \widehat{G} v) \mathrm{d} \theta \mathrm{d} \beta .
$$

The two following lemmata imply the conclusion of Theorem 1.

Lemma 1 Under the assumptions (10) and (11) $u$ is a solution of $(\mathcal{W} \widehat{\mathcal{P}})$ if and only if $u \circ \mathcal{F}$ is solution of $(\mathcal{W P})$.

Proof. We have

$$
\begin{aligned}
\widehat{a}(u, v) & =\int_{\mathcal{F}(\Omega)} \omega\left\{\nabla v \cdot \mathbf{S} \cdot \nabla u+\left[\left(\frac{1}{\omega} \nabla \omega \cdot \mathbf{S}-\mathbf{p}\right) \cdot \nabla u\right] v+\alpha u v\right\} \mathrm{d} \theta \mathrm{d} \beta \\
& =\int_{\Omega} w\left\{\nabla v(\mathcal{F}) \cdot \mathbf{A} \cdot \nabla u(\mathcal{F})+\left[\left(\frac{1}{w} \nabla w \cdot \mathbf{A}-\mathbf{b}\right) \cdot \nabla u(\mathcal{F})\right] v(\mathcal{F})+\alpha u(\mathcal{F}) v(\mathcal{F})\right\} \mathrm{d} x \mathrm{~d} y \\
& =a(u \circ \mathcal{F}, v \circ \mathcal{F})
\end{aligned}
$$

and

$$
\widehat{L}(v)=\int_{\mathcal{F}(\Omega)} \omega \widehat{G} v \mathrm{~d} \theta \mathrm{d} \beta=\int_{\Omega} G w v \circ \mathcal{F} \mathrm{d} x \mathrm{~d} y=L(v \circ \mathcal{F}) .
$$

Then, since $\mathcal{F}$ is a diffeomorfism we get the conclusion.

Lemma 2 Assume the conditions (10) and (11). Then, there exists a unique solution $u$ of the problem $(\mathcal{W} \widehat{\mathcal{P}})$.

PROOF. It is easy to see that the bilinear form $\widehat{a}$, is continuous: indeed, by Hölder inequality

$$
|\widehat{a}(u, v)| \leq \xi\|u\|_{H_{\omega}^{1}(\mathcal{F}(\Omega), \mathbf{S})}\|v\|_{H_{\omega}^{1}(\mathcal{F}(\Omega), \mathbf{S})},
$$

where

$$
\xi=\left(\frac{9}{4}+\widehat{m}_{1}\right) \sigma_{1}^{2}+\left(\frac{9}{4}+\widehat{m}_{2}\right) \sigma_{2}^{2}+\left(\frac{7}{2}+\widehat{m}_{1}+\widehat{m}_{2}\right) \sigma_{1} \sigma_{2}|\varrho|+\frac{1}{2} \mu_{1}+\frac{1}{2} \mu_{2} .
$$


Moreover, $\widehat{a}$ is coercive since

$$
\begin{aligned}
& \widehat{a}(u, u)=\int_{\mathcal{F}(\Omega)} \omega\left\{\nabla v \cdot \mathbf{S} \cdot \nabla u+\left[\left(\frac{1}{\omega} \nabla \omega \cdot \mathbf{S}-\mathbf{p}\right) \cdot \nabla u\right] v+\alpha u v\right\} \mathrm{d} \theta \mathrm{d} \beta \\
& \geq \int_{\mathcal{F}(\Omega)} \frac{\sigma_{1}^{2}}{4}(1-|\varrho|) \omega \sin ^{2}(2 \theta)\left(u_{\theta}\right)^{2}+\frac{\sigma_{2}^{2}}{4}(1-|\varrho|) \omega \sin ^{2}(2 \beta)\left(u_{\beta}\right)^{2} \\
& +\int_{\mathcal{F}(\Omega)} \frac{\mu_{1}}{4}\left(2-\left(4+\widehat{m}_{1}\right) \sin ^{2}(\theta)\right) \omega u^{2}+\frac{\mu_{2}}{4}\left(2-\left(4+\widehat{m}_{2}\right) \sin ^{2}(\beta)\right) \omega u^{2} \\
& -\int_{\mathcal{F}(\Omega)} \frac{\sigma_{1}^{2}}{2}\left(\widehat{m}_{1}^{2} \sin ^{4} \theta+\widehat{m}_{1}\left(9 \sin ^{4} \theta-7 \sin ^{2} \theta\right)+8 \sin ^{4} \theta-10 \sin ^{2} \theta+2\right) \omega u^{2} \\
& -\int_{\mathcal{F}(\Omega)} \frac{\sigma_{2}^{2}}{2}\left(\widehat{m}_{2}^{2} \sin ^{4} \beta+\widehat{m}_{2}\left(9 \sin ^{4} \beta-7 \sin ^{2} \beta\right)+8 \sin ^{4} \beta-10 \sin ^{2} \beta+2\right) \omega u^{2} \\
& -\int_{\mathcal{F}(\Omega)} \frac{\sigma_{1} \sigma_{2} \varrho}{4}\left(4 \widehat{m}_{1} \widehat{m}_{2} \sin ^{2} \theta \sin ^{2} \beta+\widehat{m}_{1}\left(10 \sin ^{2} \theta \sin ^{2} \beta-\sin ^{2} \theta\right)\right) \omega u^{2} \\
& -\int_{\mathcal{F}(\Omega)} \frac{\sigma_{1} \sigma_{2} \varrho}{4}\left(\widehat{m}_{2}\left(10 \sin ^{2} \theta \sin ^{2} \beta-\sin ^{2} \beta\right)\right) \omega u^{2} \\
& +\int_{\mathcal{F}(\Omega)} \alpha \omega u^{2}-\frac{\sigma_{1} \sigma_{2} \varrho}{4}\left(16 \sin ^{2} \theta \sin ^{2} \theta-4 \sin ^{2} \theta-4 \sin ^{2} \beta+4\right) \omega u^{2}, \\
& \widehat{a}(u, u) \geq \frac{\sigma_{1}^{2}}{4}(1-|\varrho|)\left\|\sin (2 \theta) u_{\theta}\right\|_{L_{\omega}(\mathcal{F}(\Omega))}^{2}+\frac{\sigma_{2}^{2}}{4}(1-|\varrho|)\left\|\sin (2 \beta) u_{\beta}\right\|_{L_{\omega}(\mathcal{F}(\Omega))}^{2} \\
& +\left(\alpha-\alpha_{1}\right)\|u\|_{L_{\omega}(\mathcal{F}(\Omega))}^{2}
\end{aligned}
$$

where

$$
\begin{gathered}
\alpha_{1}=\left(2+\widehat{m}_{1}\right) \frac{\mu_{1}}{4}+\left(2+\widehat{m}_{2}\right) \frac{\mu_{2}}{4}+\frac{\sigma_{1}^{2}}{2}\left(\widehat{m}_{1}^{2}+2 \widehat{m}_{1}\right)+\frac{\sigma_{2}^{2}}{2}\left(\widehat{m}_{2}^{2}+2 \widehat{m}_{2}\right) \\
+\frac{\sigma_{1} \sigma_{2}|\varrho|}{4}\left(4 \widehat{m}_{1} \widehat{m}_{2}+9 \widehat{m}_{1}+9 \widehat{m}_{2}+12\right) .
\end{gathered}
$$

Then, since

$$
\widehat{m}_{1}=m_{1}-1 \quad \text { and } \quad \widehat{m}_{2}=m_{2}-1
$$

we conclude that $\alpha_{1}=\alpha_{0}$ and from (10) we get that

$$
\widehat{a}(u, u) \geq \eta\|u\|_{H_{\omega}^{1}(\mathcal{F}(\Omega), \mathbf{S})}^{2}
$$

with

$$
\eta=\min \left\{\frac{1}{4} \sigma_{1}^{2}(1-|\varrho|), \frac{1}{4} \sigma_{2}^{2}(1-|\varrho|), \alpha-\alpha_{0}\right\} .
$$

On the other hand, the form $\widehat{L}$ is continuous since from (11)

$$
\begin{aligned}
|\widehat{L}(v)| & =\int_{0}^{\pi / 2} \int_{0}^{\pi / 2} \omega \widehat{G} v \theta \mathrm{d} \beta \\
& \leq\|\widehat{G}\|_{L_{\omega}^{2}(\mathcal{F}(\Omega))}\|v\|_{L_{\omega}^{2}(\mathcal{F}(\Omega))},
\end{aligned}
$$

because $\widehat{G} \in L_{\omega}^{2}(\mathcal{F}(\Omega))$. Finally, $\widehat{\mathcal{K}}$ is a closed convex set of $H_{\omega}^{1}(\mathcal{F}(\Omega), \mathbf{S})$. Then, by the Stampacchia Theorem (see, e.g. [6]) we conclude that the problem $(\mathcal{W} \widehat{\mathcal{P}})$ has a unique solution $u \in \widehat{\mathcal{K}}$, such that

$$
\|u\|_{H_{\omega}^{1}(\mathcal{F}(\Omega), \mathbf{S})} \leq \frac{\xi}{\eta}\|\widehat{G}\|_{L_{\omega}^{2}(\mathcal{F}(\Omega))} .
$$


This ends the proof of Lemma 2 and so of the proof of Theorem 1.

The following result shows that the weak solution satisfies, locally, the problem $(\mathcal{P})$ in almost every point. This is reduced to show that the expression $\mathcal{L}(v)=\operatorname{div}(\mathbf{A} \nabla v)+\mathbf{b} \cdot \nabla v$ makes sense in almost every point: a property clearly implied by the condition $u \in H_{l o c}^{2}(\Omega)$.

Proposition 1 Suppose the conditions of Theorem 1 and let $u$ be the weak solution of $(\mathcal{P})$. Then $u \in$ $H_{\mathrm{loc}}^{2}(\Omega)$.

PROOF. It is clear that if $u$ is a solution of $(\mathcal{W P})$ then $u$ satisfies the problem in a local weak way. More precisely, $u$ is a local weak solution of $(\mathcal{P})$ in the Brezis sense (see [5]), i.e.

$$
a(u, \eta(v-u)) \geq L(\eta(v-u)) \quad \forall v \in \mathcal{K} \quad \text { and } \quad \forall \eta \in \mathcal{D}(\Omega) .
$$

Given $\epsilon>0$ small enough, we consider the intermediate local problem

$$
\left(P_{*}\right) \begin{cases}-\mathcal{L} u_{*}+\alpha u_{*}+\gamma\left(u_{*}\right) \ni G & \text { in } \Omega_{\epsilon}^{\prime}, \\ \left(\mathbf{A} \cdot \nabla u_{*}\right) \cdot \nu=(\mathbf{A} \cdot \nabla u) \cdot \nu & \text { on } \partial \Omega_{\epsilon}^{\prime},\end{cases}
$$

where $\Omega_{\epsilon}^{\prime}=\left\{(x, y) \in \Omega: d\left((x, y), \Omega^{\prime}\right)<\epsilon\right\}$ and $\Omega^{\prime}$ is the bounded subset of $\Omega$ such that $\Omega^{\prime} \subset \Omega$. Assume $d=\operatorname{dist}\left(\partial \Omega, \Omega_{\epsilon}^{\prime}\right)>0$. Then we have

$$
\begin{aligned}
\xi \cdot \mathbf{A} \cdot \xi & =\sigma_{1}^{2} x^{2} \xi_{1}^{2}+\sigma_{2}^{2} y^{2} \xi_{2}^{2}+2 \varrho \sigma_{1} \sigma_{2} x y \xi_{1} \xi_{2} \\
& \geq \sigma_{1}^{2}(1-|\varrho|) x^{2} \xi_{1}^{2}+\sigma_{2}^{2}(1-|\varrho|) y^{2} \xi_{2}^{2} \\
& \geq \min \left\{\sigma_{1}^{2}, \sigma_{2}^{2}\right\}(1-|\varrho|) \min \left(x^{2}, y^{2}\right)\left(\xi_{1}^{2}+\xi_{2}^{2}\right) \\
& \geq \min \left\{\sigma_{1}^{2}, \sigma_{2}^{2}\right\}(1-|\varrho|)\left(\operatorname{dist}\left(\partial \Omega, \Omega_{\epsilon}^{\prime}\right)\right)^{2}|\xi|^{2} \quad \forall \xi \in \mathbb{R}^{2}-\{0\} .
\end{aligned}
$$

Then, there exists a unique weak solution of the problem $\left(\mathcal{P}_{*}\right)$. We define

$$
H^{1,+}\left(\Omega_{\epsilon}^{\prime}\right)=\left\{v \in H^{1}\left(\Omega_{\epsilon}^{\prime}\right): v \geq 0 \text { in } \Omega_{\epsilon}^{\prime}\right\} .
$$

Thanks to the regularity results of [5] the unique weak solution $u_{*}$ of problem $\left(\mathcal{P}_{*}\right)$ satisfies $u_{*} \in H^{2}\left(\Omega_{\epsilon}^{\prime}\right) \cap$ $H^{1,+}\left(\Omega_{\epsilon}^{\prime}\right)$. Then, $u_{*}$ is locally a strong solution of the problem $\left(\mathcal{P}_{*}\right)$ in the sense that

$$
\left\langle-\mathcal{L} u_{*}+\alpha u_{*}, \eta^{*}\left(v-u_{*}\right)\right\rangle_{L^{2}\left(\Omega_{\epsilon}^{\prime}\right)} \geq\left\langle G, \eta\left(v-u_{*}\right)\right\rangle_{L^{2}\left(\Omega_{\epsilon}^{\prime}\right)} \quad \forall \eta \in \mathcal{D}\left(\Omega_{\epsilon}^{\prime}\right) \text { and } \forall v \in H^{1,+}\left(\Omega_{\epsilon}^{\prime}\right) .
$$

On the other hand, it is clear that $u \in H^{1,+}\left(\Omega_{\epsilon}^{\prime}\right)$. Then, by taking $\eta^{*}=\eta w \in \mathcal{D}(\Omega)$ such that $\eta^{*} \in \mathcal{D}\left(\Omega^{\prime}\right)$, we get

$$
a\left(u-u_{*}, \eta\left(u-u_{*}\right)\right) \leq 0 .
$$

But, in general,

$$
\begin{aligned}
a(u, \eta v) & =\int_{\Omega} w\left\{\nabla(\eta v) \cdot \mathbf{A} \cdot \nabla v+\left[\left(\frac{1}{w} \nabla w \cdot \mathbf{A}-\mathbf{b}\right) \cdot \nabla v\right](\eta v)+\alpha \eta w v^{2}\right\} \mathrm{d} x \mathrm{~d} y \\
& =\int_{\Omega} \eta w \nabla v \cdot \mathbf{A} \cdot \nabla v+[(\eta \nabla w \cdot \mathbf{A}+w \nabla \eta \cdot \mathbf{A}-\eta w \mathbf{b}) \cdot \nabla v] v+\alpha \eta w v^{2} \mathrm{~d} x \mathrm{~d} y \\
& =\int_{\Omega} \eta^{*} \nabla v \cdot \mathbf{A} \cdot \nabla v+\frac{1}{2}\left(\nabla \eta^{*} \cdot \mathbf{A}-\eta^{*} \mathbf{b}\right) \cdot \nabla v^{2}+\alpha \eta^{*} v^{2} \mathrm{~d} x \mathrm{~d} y \\
& =\int_{\Omega} \eta^{*} \nabla v \cdot \mathbf{A} \cdot \nabla v+\left[\alpha \eta^{*}-\frac{1}{2} \operatorname{div}\left(\nabla \eta^{*} \cdot \mathbf{A}-\eta^{*} \mathbf{b}\right)\right] v^{2} \mathrm{~d} x \mathrm{~d} y \\
& =\int_{\Omega} \eta^{*} \nabla v \cdot \mathbf{A} \cdot \nabla v+\left[\alpha_{2} \eta^{*}-\frac{1}{2} \nabla \eta^{*} \cdot \mathbf{b}-\frac{1}{2} \operatorname{div}\left(\nabla \eta^{*} \cdot \mathbf{A}\right)\right] v^{2} \mathrm{~d} x \mathrm{~d} y
\end{aligned}
$$


where $\alpha_{2}=\alpha-\mu_{1}-\mu_{2}+2 \sigma_{1}^{2}+2 \sigma_{2}^{2}+2 \sigma_{1} \sigma_{2} \varrho \mathrm{y} \eta^{*} \in \mathcal{D}\left(\Omega_{\varepsilon}^{\prime}\right)$. Then,

$$
a(v, \eta v)=\int_{\Omega_{\varepsilon}^{\prime}} \eta^{*} \nabla v \cdot \mathbf{A} \cdot \nabla v+\left[\alpha_{2} \eta^{*}-\frac{1}{2} \nabla \eta^{*} \cdot \mathbf{b}-\frac{1}{2} \operatorname{div}\left(\nabla \eta^{*} \cdot \mathbf{A}\right)\right] v^{2} \mathrm{~d} x \mathrm{~d} y
$$

If we take $\eta^{*}$ such that

$$
\begin{aligned}
& -\operatorname{div}\left(\mathbf{A} \nabla \eta^{*}\right)-\mathbf{b} \nabla \eta^{*}+\alpha_{2} \eta^{*} \geq 0 \\
& \eta^{*}=1 \quad \text { in } \Omega^{\prime} \quad \text { and } \quad 0 \leq \eta^{*} \leq 1,
\end{aligned}
$$

then

$$
\begin{aligned}
0 & \geq a\left(u-u_{*}, \eta\left(u-u_{*}\right)\right) \\
& \geq \int_{\Omega^{\prime}} \nabla\left(u-u_{*}\right) \mathbf{A} \nabla\left(u-u_{*}\right)+\frac{1}{2} \alpha_{2}\left(u-u_{*}\right)^{2} \\
& \geq \min \left\{\sigma_{1}^{2}(1-|\varrho|), \sigma_{2}^{2}(1-|\varrho|), \frac{1}{2} \alpha_{2}\right\}\left|u-u_{*}\right|_{H^{1}\left(\Omega^{\prime}\right)}^{2}
\end{aligned}
$$

Finally, we deduce that $u=u_{*}$ on $\Omega^{\prime}$, and so $u \in H^{2}\left(\Omega^{\prime}\right)$.

Before to study the localization of the free boundary it is useful to obtain some a priori $L^{\infty}(\Omega)$ estimates on the solution.

Proposition 2 Assume the conditions of Theorem 1. Let $u$ be the weak solution of $(\mathcal{P})$. Suppose, additionally, that

$$
w_{0} G^{+} \in L^{\infty}(\Omega)
$$

with

$$
w_{0}(x, y)=\left(1+x^{2}\right)^{-\frac{m_{1}+1}{8}}\left(1+y^{2}\right)^{-\frac{m_{2}+1}{8}}
$$

Then we have

$$
0 \leq w_{0} u \leq \frac{1}{\alpha-\alpha_{0}}\left|G^{+} w_{0}\right|_{L^{\infty}(\Omega)}
$$

ProOF. Let $k=\frac{1}{\alpha-\alpha_{0}}\left\|G^{+} w_{0}\right\|_{L^{\infty}(\Omega)}$ and consider $v_{0}=u-\left(u-k w_{0}^{-1}\right)^{+}$as a test function. Then we have $v_{0} \in H_{w}^{1}(\Omega, \mathbf{A})$. Let $u$ be a solution of the problem, then

$$
a\left(u, u-v_{0}\right) \leq \int_{\Omega} w G\left(u-v_{0}\right)
$$

i.e.

$$
a\left(u,\left(u-k w_{0}^{-1}\right)^{+}\right) \leq \int_{\Omega} w G\left(u-k w_{0}^{-1}\right)^{+} .
$$

Then

$$
\begin{aligned}
a\left(u-k w_{0}^{-1},\left(u-k w_{0}^{-1}\right)^{+}\right) \leq & -k\left\langle-\mathcal{L}\left(w_{0}^{-1}\right)+\alpha w_{0}^{-1}, w\left(u-k w_{0}^{-1}\right)^{+}\right\rangle_{L^{2}(\Omega)} \\
& +\int_{\Omega} w G\left(u-k w_{0}^{-1}\right)^{+} \\
\leq & \int_{\Omega} w\left[G-k\left(-\mathcal{L}\left(w_{0}^{-1}\right)+\alpha w_{0}^{-1}\right)\right]\left(u-k w_{0}^{-1}\right)^{+} .
\end{aligned}
$$


Let us evaluate $-\mathcal{L}\left(w_{0}^{-1}\right)+\alpha w_{0}^{-1}$ :

$$
\begin{aligned}
w_{0}\left(-\mathcal{L}\left(w_{0}^{-1}\right)+\alpha w_{0}^{-1}\right)=\alpha & -\frac{\sigma_{1}^{2}}{16}\left(m_{1}+1\right) \frac{x^{2}}{1+x^{2}}\left(4+\left(m_{1}-7\right) \frac{x^{2}}{1+x^{2}}\right) \\
& -\frac{\sigma_{2}^{2}}{16}\left(m_{2}+1\right) \frac{y^{2}}{1+y^{2}}\left(4+\left(m_{2}-7\right) \frac{y^{2}}{1+y^{2}}\right) \\
& -\frac{\sigma_{1} \sigma_{2} \varrho}{8}\left(m_{1}+1\right)\left(m_{2}+1\right) \frac{x^{2} y^{2}}{\left(1+x^{2}\right)\left(1+y^{2}\right)} \\
& -\frac{\mu_{1}}{4}\left(m_{1}+1\right) \frac{x^{2}}{1+x^{2}}-\frac{\mu_{2}}{4}\left(m_{2}+1\right) \frac{y^{2}}{1+y^{2}} \\
\geq \alpha & -\frac{\sigma_{1}^{2}}{16}\left(m_{1}+1\right)\left(4+\left(m_{1}-7\right)\right)-\frac{\sigma_{2}^{2}}{16}\left(m_{2}+1\right)\left(4+\left(m_{2}-7\right)\right) \\
& -\frac{\sigma_{1} \sigma_{2}|\varrho|}{8}\left(m_{1}+1\right)\left(m_{2}+1\right)-\frac{\mu_{1}}{4}\left(m_{1}+1\right)-\frac{\mu_{2}}{4}\left(m_{2}+1\right) \\
\geq \alpha & -\alpha_{0}
\end{aligned}
$$

thus

$$
-k\left(-\mathcal{L}\left(w_{0}^{-1}\right)+\alpha w_{0}^{-1}\right) \leq-k\left(\alpha-\alpha_{0}\right) w_{0}^{-1} .
$$

In consequence, since $\alpha-\alpha_{0}>0$, we get

$$
\begin{aligned}
a\left(u-k w_{0}^{-1},\left(u-k w_{0}^{-1}\right)^{+}\right) & \left.\leq \int_{\Omega} w\left(G-k\left(\alpha-\alpha_{0}\right) w_{0}^{-1}\right)\right)\left(u-k w_{0}^{-1}\right)^{+} \\
& \left.\leq \int_{\Omega} w\left(G-\left\|G^{+} w_{0}\right\|_{L^{\infty}} w_{0}^{-1}\right)\right)\left(u-k w_{0}^{-1}\right)^{+} \\
& \leq 0 .
\end{aligned}
$$

Since $a$ is coercive (use that $\widehat{a}$ is coercive), we get that $\left(u-k w_{0}^{-1}\right)^{+} \equiv 0$ en $\Omega$ which implies the conclusion.

\section{Localizing the free boundary}

\subsection{The main result}

We are interested now in the study of the coincidence set defined as the set of points $(x, y) \in \Omega$ where the solution coincides with the obstacle. In the case of the "new" formulation $u=v-h$ the obstacle is reduced to the function zero, and so, the domain of coincidence is the domain of points where $u$ vanishes. As we can not give an explicit formula for this set (see e.g. [20]) we shall give some a priori estimates on its localization. For a given general function $\Psi: \Omega \rightarrow \mathbb{R}^{+}$we define the null and positive sets associated to $\Psi$ as follows:

$$
\begin{aligned}
& N(\Psi) \doteq \text { null set of } \Psi \doteq\{(x, y) \in \bar{\Omega}: \quad \Psi(x, y)=0\}, \\
& S^{+}(\Psi) \doteq \text { positive set of } \Psi \doteq \overline{=\{(x, y) \in \Omega: \quad \Psi(x, y)>0\}} .
\end{aligned}
$$

We note that $\Omega=N(u) \cup S^{+}(u)$ but we do not know, a priori, if the coincidence set $N(u)$ is empty or not. Obviously it depends on the data $G(x, y)$. Our next result states that this set is not empty if $G(x, y)$ is "sufficiently negative" in some subset of $\Omega$ "big enough". In fact, in this case we shall get also an estimate on its location.

Our method of proof is inspired on the, so called, method of local supersolutions, (see a previous exposition in Díaz [11]). One of the main difficulties in implementing such method to our problem comes 
from the fact that the coefficients of operator $\mathcal{L}(u)$ depend strongly of the different points $(x, y) \in \Omega$. To carry out our study we introduce the distance $\widetilde{d}$ on $\Omega$ given by

$$
\widetilde{d}\left(\left(x_{0}, y_{0}\right),(x, y)\right)=\sqrt{\left(\log \left(\frac{x}{x_{0}}\right)\right)^{2}+\left(\log \left(\frac{y}{y_{0}}\right)\right)^{2}} .
$$

Motivated by the special structure of the coefficient of the operator $\mathcal{L}(u)$ we introduce the auxiliary function

$$
\begin{aligned}
\epsilon(x, y)=\{ & \left.\frac{1}{8}\left(\sigma_{1}^{2}+\sigma_{2}^{2}\right)(2-|\varrho|)\left(m_{1}+m_{2}+2\right)^{2}+\left(\left|\mu_{1}-\sigma_{1}^{2}\right|+\left|\mu_{2}-\sigma_{2}^{2}\right|\right)\left(m_{1}+m_{2}+2\right)\right\} \\
& \mathrm{e}^{2} \frac{1}{\alpha-\alpha_{0}}\left|G^{+} w_{0}\right|_{L^{\infty}(\omega)} w_{0}^{-1}
\end{aligned}
$$

where $w_{0}(x, y)$ is given by (13).

Assume now that $\Gamma_{0} \doteq\{(x, y): G(x, y)=0\}$ is a monotone graph and that, for any $(x, y) \in \Gamma_{0}$ and $h>0$ we have $(x, y+h) \in S^{+}(-G)$. Let $T_{a, b}$ be the application defined by

$$
\begin{aligned}
T_{a, b}: \mathbb{R}^{2} & \rightarrow \mathbb{R}^{2} \\
& (x, y) \rightarrow\left(x \mathrm{e}^{a}, y \mathrm{e}^{b}\right)
\end{aligned}
$$

then

$$
T_{a, b}^{-1}=T_{-a,-b}
$$

Let $h_{1}, h_{2}$ and $k_{1}$ in $\mathbb{R}^{2}$, we can note that

$$
\text { if } \quad\left(x-h_{1}, y-k_{1}\right) \in S^{+}(-G) \quad \text { and } \quad\left(x+h_{2}, y-k_{1}\right) \in S^{+}(-G),
$$

then

$$
\forall k_{2} \geq 0 \quad\left(x-h_{1}, x+h_{2}\right) \times\left(y-k_{1}, y+k_{2}\right) \subset S^{+}(-G) .
$$

Theorem 2 Let $u$ be solution of the problem $(\mathcal{P})$ and assume that the set

$$
S^{+}(-G-\epsilon) \doteq \overline{=\{(x, y) \in \Omega: G(x, y) \leq-\epsilon(x, y)\}}
$$

is not empty. Then the coincidence set $N(u)$ satisfies that

$$
N(u) \supset\left\{(x, y) \in T_{R_{0}, R_{0}}\left(S^{+}(-G-\epsilon)\right) \bigcap T_{-R_{0}, R_{0}}\left(S^{+}(-G-\epsilon)\right): \widetilde{d}((x, y), \partial \Omega) \geq R_{0}\right\},
$$

where $R_{0}=\frac{4}{m_{1}+m_{2}+2}$.

Proof. We consider the set

$$
\Omega_{R} \doteq\left\{(x, y) \in T_{R, R}\left(S^{+}(-G-\epsilon)\right) \bigcap T_{-R, R}\left(S^{+}(-G-\epsilon)\right): 2 \widetilde{d}((x, y), \partial \Omega) \geq R\right\} .
$$

Let $\left(x_{0}, y_{0}\right) \in \Omega_{R}$. We define

$$
\widetilde{B}_{R}\left(x_{0}, y_{0}\right) \doteq\left\{(x, y) \in \Omega:\left(\log \left(\frac{x}{x_{0}}\right)\right)^{2}+\left(\log \left(\frac{y}{y_{0}}\right)\right)^{2}<R^{2}\right\} .
$$

We point out that

$$
\widetilde{B}_{R}\left(x_{0}, y_{0}\right) \subset\left(x_{0} \mathrm{e}^{-R}, x_{0} \mathrm{e}^{R}\right) \times\left(y_{0} \mathrm{e}^{-R}, y_{0} \mathrm{e}^{R}\right)
$$


and that

$$
G\left(x_{0} \mathrm{e}^{-R}, y_{0} \mathrm{e}^{-R}\right) \leq \epsilon \quad \text { and } \quad G\left(x_{0} \mathrm{e}^{R}, y_{0} \mathrm{e}^{-R}\right) \leq \epsilon
$$

since

$$
\forall(x, y) \in \widetilde{B}_{R}\left(x_{0}, y_{0}\right) \quad G(x, y) \leq \epsilon .
$$

We shall get our conclusion by construing a local supersolution $\bar{u}\left(x, y: x_{0}, y_{0}\right)$ defined on $\widetilde{B}_{R}\left(x_{0}, y_{0}\right)$, for any $\left(x_{0}, y_{0}\right) \in \Omega_{R}$. We search such function in the class of radially symmetric functions

$$
\bar{u}\left(x, y: x_{0}, y_{0}\right)=\eta(r),
$$

where $\eta>0, \eta^{\prime}>0$ and $\eta^{\prime \prime}>0 \forall r>0$, which will be determined later, and with $r=\widetilde{d}\left((x, y) ;\left(x_{0}, y_{0}\right)\right)$. We have

$$
\begin{aligned}
\mathcal{L} \eta=\{ & \sigma_{1}^{2} \frac{\left(\log (x)-\log \left(x_{0}\right)\right)^{2}}{r^{2}}+\sigma_{2}^{2} \frac{\left(\log (y)-\log \left(y_{0}\right)\right)^{2}}{r^{2}} \\
& \left.+2 \sigma_{1} \sigma_{2} \varrho \frac{\left(\log (x)-\log \left(x_{0}\right)\right)\left(\log (y)-\log \left(y_{0}\right)\right)}{r^{2}}\right\} \eta^{\prime \prime} \\
& +\left\{\sigma_{1}^{2}+\sigma_{2}^{2}-\left(\sigma_{1}^{2} \frac{\left(\log (x)-\log \left(x_{0}\right)\right)^{2}}{r^{2}}+\sigma_{2}^{2} \frac{\left(\log (y)-\log \left(y_{0}\right)\right)^{2}}{r^{2}}\right.\right. \\
& +2 \sigma_{1} \sigma_{2} \varrho \frac{\left(\log (x)-\log \left(x_{0}\right)\right)\left(\log (y)-\log \left(y_{0}\right)\right)}{r^{2}} \\
& \left.\left.+\left(\mu_{1}-\sigma_{1}^{2}\right)\left(\log (x)-\log \left(x_{0}\right)\right)+\left(\mu_{2}-\sigma_{2}^{2}\right)\left(\log (y)-\log \left(y_{0}\right)\right)\right)\right\} \frac{\eta^{\prime}}{r} .
\end{aligned}
$$

Then,

$$
\begin{aligned}
\mathcal{L} \eta \leq & \left\{\sigma_{1}^{2}(1-|\varrho|) \frac{\left(\log (x)-\log \left(x_{0}\right)\right)^{2}}{r^{2}}+\sigma_{2}^{2}(1-|\varrho|) \frac{\left(\log (y)-\log \left(y_{0}\right)\right)^{2}}{r^{2}}\right\} \eta^{\prime \prime} \\
& +\left\{\sigma_{1}^{2}+\sigma_{2}^{2}-\left(\sigma_{1}^{2}(1+|\varrho|) \frac{\left(\log (x)-\log \left(x_{0}\right)\right)^{2}}{r^{2}}+\sigma_{2}^{2}(1+|\varrho|) \frac{\left(\log (y)-\log \left(y_{0}\right)\right)^{2}}{r^{2}}\right.\right. \\
+ & \left.\left.\left(\mu_{1}-\sigma_{1}^{2}\right)\left(\log (x)-\log \left(x_{0}\right)\right)+\left(\mu_{2}-\sigma_{2}^{2}\right)\left(\log (y)-\log \left(y_{0}\right)\right)\right)\right\} \frac{\eta^{\prime}}{r} \\
\leq & \left(\sigma_{1}^{2}+\sigma_{2}^{2}\right)(1-|\varrho|) \eta^{\prime \prime}+\left\{\sigma_{1}^{2}+\sigma_{2}^{2}-\min \left\{\sigma_{1}^{2}, \sigma_{2}^{2}\right\}(1+|\varrho|)+2 r\left(\left|\mu_{1}-\sigma_{1}^{2}\right|+\left|\mu_{2}-\sigma_{2}^{2}\right|\right)\right\} \frac{\eta^{\prime}}{r} \\
\leq & \left(\sigma_{1}^{2}+\sigma_{2}^{2}\right)(1-|\varrho|) \eta^{\prime \prime}+\left\{\sigma_{1}^{2}+\sigma_{2}^{2}+2 r\left(\left|\mu_{1}-\sigma_{1}^{2}\right|+\left|\mu_{2}-\sigma_{2}^{2}\right|\right)\right\} \frac{\eta^{\prime}}{r} .
\end{aligned}
$$

Thus, for $r \in(0, R)$ we get that

$$
\mathcal{L} \eta \leq\left(\sigma_{1}^{2}+\sigma_{2}^{2}\right)(1-|\varrho|) \eta^{\prime \prime}+\left\{\sigma_{1}^{2}+\sigma_{2}^{2}+2 R\left(\left|\mu_{1}-\sigma_{1}^{2}\right|+\left|\mu_{2}-\sigma_{2}^{2}\right|\right)\right\} \frac{\eta^{\prime}}{r} .
$$

Finally

$$
-\mathcal{L} \eta+\alpha \eta \geq-\left(\sigma_{1}^{2}+\sigma_{2}^{2}\right)(1-|\varrho|) \eta^{\prime \prime}-\left\{\sigma_{1}^{2}+\sigma_{2}^{2}+2 R\left(\left|\mu_{1}-\sigma_{1}^{2}\right|+\left|\mu_{2}-\sigma_{2}^{2}\right|\right)\right\} \frac{\eta^{\prime}}{r} .
$$

On the other hand, it is easy to check that given $\epsilon_{0}>0$, the solution of the problem

$$
\left\{\begin{array}{l}
-\left(\sigma_{1}^{2}+\sigma_{2}^{2}\right)(1-|\varrho|) \eta^{\prime \prime}-\left\{\sigma_{1}^{2}+\sigma_{2}^{2}+2 R\left(\left|\mu_{1}-\sigma_{1}^{2}\right|+\left|\mu_{2}-\sigma_{2}^{2}\right|\right)\right\} \frac{\eta^{\prime}}{r}+\mathcal{B}(\eta) \ni-\epsilon_{0} \quad \text { in }(0, R) \\
\eta(0)=\eta^{\prime}(0)=0
\end{array}\right.
$$


is given by

The condition

$$
\eta(r)=\frac{\epsilon_{0}}{2\left(\sigma_{1}^{2}+\sigma_{2}^{2}\right)(2-|\varrho|)+4 R\left(\left|\mu_{1}-\sigma_{1}^{2}\right|+\left|\mu_{2}-\sigma_{2}^{2}\right|\right)} r^{2} .
$$

$$
\epsilon_{0} \leq-G \quad \text { in } \quad \widetilde{B}_{R}\left(x_{0}, y_{0}\right) \quad \text { and } \quad \eta(R) \geq\left. u\right|_{\partial B_{R}\left(X_{0}\right)}
$$

or, equivalently,

$$
\eta(R)=\frac{\epsilon_{0}}{2\left(\sigma_{1}^{2}+\sigma_{2}^{2}\right)(2-|\varrho|)+4 R\left(\left|\mu_{1}-\sigma_{1}^{2}\right|+\left|\mu_{2}-\sigma_{2}^{2}\right|\right)} R^{2} \geq \sup _{\partial B_{R}} K w^{-l}(x, y)
$$

and

$$
\epsilon_{0}=\inf _{B_{R}\left(X_{0}\right)}\{\epsilon(x, y)\},
$$

implies that $\eta$ is a supersolution of $(\mathcal{P})$. Then $\eta$ is also a supersolution of $(\mathcal{P})$ in $\widetilde{B}_{R}\left(x_{0}, y_{0}\right)$. Finally, the choice of $\epsilon(x, y)$ and $R=\frac{4}{m_{1}+m_{2}+2}$ leads to the option $u(x, y)=0$ for any $(x, y) \in \Omega_{R_{0}}$.

Remark 1 To get some special conclusions obtained via Theorem 2 for several special type examples of data $G(x, y)$, the reader can consult [12] (see also [14]).

Remark 2 An evolution problem quite related to the deterministic problem considered in this paper was the main object of the paper [10].

\subsection{A simple example}

By using other different techniques, we consider consider now an illustrative example (suggested to us by G. Díaz) to understand the process of how to develop such a criterion for an optimal action. We consider a simple onedimensional model where the environmental benefits at time $t$ are given by $\mathcal{X}(t)$ solution of

$$
\left\{\begin{array}{l}
\mathrm{d} \mathcal{X}(t)=\sqrt{2} \sigma \mathcal{X}(t) \mathrm{d} \mathcal{B}(t), \quad t>0, \quad(\sigma>0) \\
\mathcal{X}(0)=x \in \mathbb{R}_{+} .
\end{array}\right.
$$

The alternative project is assumed to have a constant benefit $\mathcal{Y}(t)=c, t>0$. It is worth considering the logarithmic scale $\widehat{\mathcal{X}}(t)=\log \mathcal{X}(t)$. Using the Ito theorem we get

$$
\mathrm{d} \widehat{\mathcal{X}}(t)=\frac{1}{\mathcal{X}(t)} \mathrm{d} \mathcal{X}(t)-\frac{1}{2(\mathcal{X}(t))^{2}} 2 \sigma^{2}(\mathcal{X}(t))^{2} \mathrm{~d} t
$$

i.e.

or equivalently

$$
\left\{\begin{array}{l}
\mathrm{d} \widehat{\mathcal{X}}(t)=-\sigma^{2} \mathrm{~d} t+\sqrt{2} \sigma \mathrm{d} \mathcal{B}(t), \quad t>0 \\
\widehat{\mathcal{X}}(0)=\log x \doteq \widehat{x} \in \mathbb{R}
\end{array}\right.
$$

$$
\widehat{\mathcal{X}}(t) \sim \mathcal{N}\left(\widehat{x}-\sigma^{2} t, \sqrt{2 t} \sigma\right), \quad t>0 .
$$

Following the same philosophy, we introduce the logarithmic scale in the value that involves the control time $\mathrm{T} \in \mathbb{R}_{+}$

$$
\begin{aligned}
\mathcal{J}(\widehat{x} ; \mathrm{T})= & \mathbb{E}\left[\int_{0}^{+\infty} \mathrm{e}^{-\alpha s}\left[\mathbb{1}_{] 0, \mathrm{~T}[}(s) \log (\mathcal{X}(s))+\mathbb{I}_{] \mathrm{T},+\infty[}(s) c\right] \mathrm{d} s\right] \\
= & \frac{1}{\alpha}\left\{\widehat{x}\left(1-\mathrm{e}^{-\alpha \mathrm{T}}\right)-\sigma^{2}\left(\frac{1-\mathrm{e}^{-\alpha \mathrm{T}}}{\alpha}-\mathrm{Te}^{-\alpha \mathrm{T}}\right)+\widehat{c} \mathrm{e}^{-\alpha \mathrm{T}}\right\} \\
& +\sqrt{2} \sigma \mathbb{E}\left[\int_{0}^{\mathrm{T}} \mathrm{e}^{-\alpha s} \mathcal{B}(s) \mathrm{d} s\right]
\end{aligned}
$$


where $\widehat{c} \doteq \log c$ and $\alpha>0$ is the discount term .

Clearly the function

$$
\widehat{x} \mapsto \mathcal{J}(\widehat{x} ; \mathrm{T})
$$

is linear increasing and affine, so, the function optimizing the logarithmic cost

$$
\widehat{\mathcal{V}}(\widehat{x})=\sup _{\mathrm{T} \in \mathbb{R}_{+}} \mathcal{J}(\widehat{x} ; \mathrm{T}), \quad \widehat{x} \in \mathbb{R},
$$

is convex.

Then, from its semicontinuity to the right it is locally Lipschitz on $\mathbb{R}$. Indeed, thanks to the existence of $\mathrm{T}_{\varepsilon}^{\widehat{x}_{1}}>0$ such that

$$
\widehat{\mathcal{V}}\left(\widehat{x}_{1}\right)-\widehat{\mathcal{V}}\left(\widehat{x}_{2}\right) \leq \mathcal{J}\left(\widehat{x}_{1} ; \mathrm{T}_{\varepsilon}^{\widehat{x}_{1}}\right)-\mathcal{J}\left(\widehat{x}_{2} ; \mathrm{T}_{\varepsilon}^{\widehat{x}_{1}}\right)+\varepsilon \leq \frac{1-e^{-\alpha \mathrm{T}_{\varepsilon}^{\widehat{x}_{1}}}}{\alpha}\left|\widehat{x}_{1}-\widehat{x}_{2}\right|+\varepsilon \quad(\varepsilon>0),
$$

the function $\widehat{\mathcal{V}}$ satisfies

$$
\left|\widehat{\mathcal{V}}\left(\widehat{x}_{1}\right)-\widehat{\mathcal{V}}\left(\widehat{x}_{2}\right)\right| \leq \frac{1}{\alpha}\left|\widehat{x}_{1}-\widehat{x}_{2}\right|, \quad \widehat{x}_{1}, \widehat{x}_{2} \in \mathbb{R}
$$

In consequence, $\widehat{\mathcal{V}}$ increases, near the infinite, at most linearly. More precisely,

$$
\widehat{\mathcal{V}}(\widehat{x}) \geq \liminf _{\mathrm{T} \rightarrow 0} \mathcal{J}(\widehat{x} ; \mathrm{T})=\frac{\widehat{c}}{\alpha}, \quad \widehat{x} \in \mathbb{R}^{2} .
$$

Moreover

$$
\frac{1-\mathrm{e}^{-\alpha \mathrm{T}}}{\alpha} \leq \lim _{\widehat{x} \rightarrow+\infty} \frac{\mathcal{J}(\widehat{x} ; \mathrm{T})}{\widehat{x}} \leq \liminf _{\widehat{x} \rightarrow+\infty} \frac{\widehat{\mathcal{V}}(\widehat{x})}{\widehat{x}} \leq \limsup _{\widehat{x} \rightarrow+\infty} \frac{\widehat{\mathcal{V}}(\widehat{x})}{\widehat{x}} \leq \lim _{\widehat{x} \rightarrow+\infty} \frac{\mathcal{J}\left(\widehat{x} ; \mathrm{T}_{\varepsilon}^{\widehat{x}}\right)+\varepsilon}{\widehat{x}} \leq \frac{1}{\alpha},
$$

for a suitable $\mathrm{T}_{\varepsilon}^{\widehat{x}}, \varepsilon>0$ small enough. Then, as, $\mathrm{T}$ is arbitrary we get

$$
\lim _{\widehat{x} \rightarrow+\infty} \frac{\widehat{\mathcal{V}}(\widehat{x})}{\widehat{x}}=\frac{1}{\alpha}
$$

By means of the Dynamics Programming

$$
\begin{aligned}
\widehat{\mathcal{V}}(\widehat{x}) & \geq \mathcal{J}(\widehat{x} ; \mathrm{T}+h)=\mathbb{E}\left[\int_{0}^{h} \mathrm{e}^{-\alpha s} \widehat{\mathcal{X}}(s) \mathrm{d} s+\int_{h}^{\mathrm{T}+h} \mathrm{e}^{-\alpha s} \widehat{\mathcal{X}}(s) \mathrm{d} s\right]+\int_{\mathrm{T}+h}^{+\infty} \widehat{c} \mathrm{e}^{-\alpha s} \mathrm{~d} s \\
& =\mathbb{E}\left[\int_{0}^{h} \mathrm{e}^{-\alpha s} \widehat{\mathcal{X}}(s) \mathrm{d} s\right]+\mathrm{e}^{-\alpha h}\left(\mathbb{E}\left[\int_{0}^{\mathrm{T}} \mathrm{e}^{-\alpha \hat{s}} \widehat{\mathcal{X}}(\hat{s}+h) \mathrm{d} \hat{s}\right]+\int_{\mathrm{T}}^{+\infty} \widehat{c} \mathrm{e}^{-\alpha s} \mathrm{~d} \hat{s}\right) \\
& =\mathbb{E}\left[\int_{0}^{h} \mathrm{e}^{-\alpha s} \widehat{\mathcal{X}}(s) \mathrm{d} s\right]+\mathrm{e}^{-\alpha h} \mathcal{J}(\widehat{\mathcal{X}}(h) ; \mathrm{T}), \quad \mathrm{T} \in \mathbb{R}_{+},
\end{aligned}
$$

i.e.

$$
\widehat{\mathcal{V}}(\widehat{x}) \geq \mathbb{E}\left[\int_{0}^{h} \mathrm{e}^{-\alpha s} \widehat{\mathcal{X}}(s) \mathrm{d} s\right]+\mathrm{e}^{-\alpha h} \mathbb{E}[\widehat{\mathcal{V}}(\widehat{\mathcal{X}}(h))], \quad \widehat{x} \in \mathbb{R} .
$$

Then, some classical arguments, we can show that $\widehat{\mathcal{V}}$ is a viscosity continuous solution of the nonlinear ordinary differential equation

$$
\min \left\{-\sigma^{2} \widehat{\mathcal{V}}^{\prime \prime}+\sigma^{2} \widehat{\mathcal{V}}^{\prime}+\alpha \widehat{\mathcal{V}}-\widehat{x}, \widehat{\mathcal{V}}-\frac{\widehat{c}}{\alpha}\right\}=0 \quad \text { in } \mathbb{R}
$$


in fact, by the Aleksandrov theorem (see [8, Theorem A.2]) the function $\mathcal{V}$ is twice differentiable in almost any point. So, we get that

$$
\min \left\{-\sigma^{2} \widehat{\mathcal{V}}^{\prime \prime}+\sigma^{2} \widehat{\mathcal{V}}^{\prime}+\alpha \widehat{\mathcal{V}}-\widehat{x}, \widehat{\mathcal{V}}-\frac{\widehat{c}}{\alpha}\right\}=0 \quad \text { a.e. } \widehat{x} \in \mathbb{R} .
$$

Since $\widehat{\mathcal{V}}(\cdot)$ is increasing and convex we can ensure the existence a free boundary $\left\{\widehat{x}_{f}\right\}$ separating the coincidence region

$$
\left.\left.\left[\widehat{\mathcal{V}}=\frac{\widehat{c}}{\alpha}\right]=\right]-\infty, \widehat{x}_{f}\right]
$$

from the continuation region

$$
\left.\left[\widehat{\mathcal{V}}>\frac{\widehat{c}}{\alpha}\right]=\right] \widehat{x}_{f},+\infty[
$$

characterized by $\widehat{\mathcal{V}}^{\prime}\left(\widehat{x}_{f}^{-}\right)=0$. If we assume that the continuation region is the whole space of zero characteristic

$$
\lambda^{ \pm}=\frac{1}{2}\left(1 \pm \sqrt{1+4 \frac{\alpha}{\sigma^{2}}}\right),
$$

from the relative differential operator we deduce the representation

$$
\mathcal{W}(\widehat{x})=C \mathrm{e}^{\lambda^{+} \widehat{x}}+D \mathrm{e}^{\lambda^{-} \widehat{x}}+\frac{\widehat{x}}{\alpha}-\frac{\sigma^{2}}{\alpha^{2}}, \quad \widehat{x} \in \mathbb{R} \quad(C, D \in \mathbb{R})
$$

for any solution. Then, from

$$
\lambda^{+}>1 \text { and } \lambda^{-}<0
$$

and the increasing assumption (15) we get that $C=0$. Moreover, by the lower bound

$$
\widehat{\mathcal{V}}(\widehat{x}) \geq \frac{\widehat{c}}{\alpha}
$$

this representation is valid only in the continuation region $] \widehat{x}_{f},+\infty[$. In consequence we have

$$
\mathcal{W}(\widehat{x})=\delta e^{\lambda^{-}\left(\widehat{x}-\widehat{x}_{f}\right)}+\frac{\widehat{x}}{\alpha}-\frac{\sigma^{2}}{\alpha^{2}}, \quad \widehat{x} \geq \widehat{x}_{f} \quad(\delta \in \mathbb{R}) .
$$

From the uniqueness of the viscosity solution of the problem

$$
\begin{cases}-\sigma^{2} \mathcal{W}^{\prime \prime}+\sigma^{2} \mathcal{W}^{\prime}+\alpha \mathcal{W}=\widehat{x} & \text { en }] \widehat{x}_{f},+\infty[, \\ \mathcal{W}\left(\widehat{x}_{f}\right)=\frac{\widehat{c}}{\alpha} & \text { and } \quad \lim _{\widehat{x} \rightarrow+\infty} \frac{\mathcal{W}(\widehat{x})}{\widehat{x}}=\frac{1}{\alpha}\end{cases}
$$

and the results on viscosity solutions we get that

$$
\widehat{\mathcal{V}}(\widehat{x})=\frac{1}{\alpha}\left(\left(\widehat{c}-\widehat{x}_{f}+\frac{\sigma^{2}}{\alpha}\right) \mathrm{e}^{\lambda^{-(}\left(\widehat{x}-\widehat{x}_{f}\right)}+\widehat{x}-\frac{\sigma^{2}}{\alpha}\right), \quad \widehat{x} \geq \widehat{x}_{f} .
$$

Then, from the uniqueness and complementary regularity for the problem

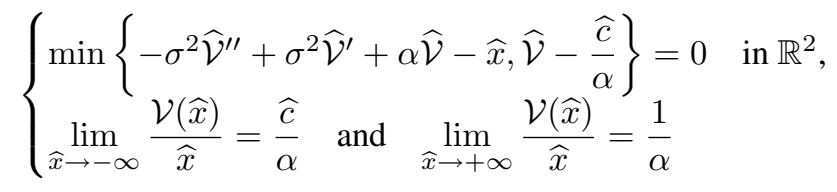


(see [3]) we deduce the property $\widehat{\mathcal{V}} \in \mathcal{C}^{1,1}(\mathbb{R})$, and so we get

$$
0=\widehat{\mathcal{V}}_{\prime}\left(\widehat{x}_{f}\right)=\frac{1}{\alpha}\left(\left(\widehat{c}-\widehat{x}_{f}+\frac{\sigma^{2}}{\alpha}\right) \lambda^{-}+1\right) \Leftrightarrow \widehat{x}_{f}=\widehat{c}+\frac{\sigma^{2}}{\alpha}+\frac{1}{\lambda^{-}} .
$$

Thus, the solution of the problem is

$$
\widehat{\mathcal{V}}(\widehat{x})= \begin{cases}\frac{\widehat{c}}{\alpha}, & 0<\widehat{x} \leq \widehat{x}_{f}, \\ \frac{1}{\alpha}\left(\left(\widehat{c}-\widehat{x}_{f}+\frac{\sigma^{2}}{\alpha}\right) \mathrm{e}^{\lambda^{-}\left(\widehat{x}-\widehat{x}_{f}\right)}+\widehat{x}-\frac{\sigma^{2}}{\alpha}\right), & \widehat{x}_{f}<\widehat{x}\end{cases}
$$

Note that since

$$
\lim _{\sigma \rightarrow 0} \lambda^{-}=-\infty
$$

the free boundary is attaint in the point.

$$
\widehat{x}_{f}^{0}=\widehat{c} \text {. }
$$

So, in absence of risk the solution is

$$
\widehat{\mathcal{V}}^{0}(\widehat{x})= \begin{cases}\frac{\widehat{c}}{\alpha}, & 0<\widehat{x} \leq \widehat{c} \\ \frac{\widehat{x}}{\alpha}, & \widehat{c}<\widehat{x}\end{cases}
$$

Acknowledgement. The authors thank to G .Díaz and J. A. Sheinkman the comments and suggestions received during the preparation of this work. The research of J. I. Díaz was partially supported by the projects MTM2005-03463 of the DGISGP (Spain) and CCG07-UCM/ESP-2787 of the CAM and UCM

\section{References}

[1] Akerlof, G. A. (1998), The Market for Lemons: Qualitative Uncertainty and the Market Mechanism, Quarterly Journal of Economics, 84, 488-500.

[2] Bellman, R. (1957), Dynamic Programming. Academic Press, London.

[3] Bensoussan, A. AND LiOns, J. L., (1978). Application des inégalités variationnelles en control stochastique. Dunod, Paris.

[4] Bermudez, A., Moreno, C. And Sanmartin, A., (1997). Resolución numérica de un problema de valor óptimo de una opción. In Actas de la Jornada Científica en homenaje a A. Valle , Caraballo, T., et al Eds, Publicaciones de la Universidad de Sevilla.

[5] Brezis, H., (1972). Problèmes Unilateraux. J. Math. Pures et Appl., 51, 1-168.

[6] BrezIs, H., (1983). Analyse fonctionnelle. Masson, Paris, .

[7] Brezis, H. And Friedman, A., (1976). Estimates on the support of the solutions of parabolic variational inequalities. Illinois J. Math, 20, 82-97.

[8] Crandall, M. G., Ishit, H. And Lions, P. L., (1992). User's guide to viscosity solutions of second order partial differential equations. Bull. Amer. Math. Soc., 27, 1-67.

[9] DíAz, G., (1985). Acción óptima en una ecuación de la Programación Dinámica. Rev. R. Acad. Cienc. Exactas Fis. Nat., LXXIX, 89-105.

[10] Díaz, G., Díaz, J. I., FAghloumi C.,, (2007). On an evolution problem associated to the modelling of incertitude into the Environment. Nonlinear Analysis: Real World Applications, 8, 399-404. 
[11] DíAZ, J. I., (1985). Nonlinear partial differential equations and free boundaries. 106, Pitman, London.

[12] Díaz, J. I., Faghloumi, C., (2002). Analysis of a nonlinear elliptic problem arising in the study of policies on projects altering the environment, Applied Math. and Optimization, 45, 251-267.

[13] DiXit, A. K. And Pindyck, R. S., (1994). Investment under Uncertainly . Princeton University Press, Princeton.

[14] Faghloumi, C., (2004). Modelizacion y tratamiento de algunos problemas de Medio Ambiente. Ph. D.,thesis, Universidad Complutense de Madrid, Madrid.

[15] Fleming, W. H. AND Rishel, R. (1975). Deterministic and stochastic optimal control, Springer-Verlag, New York.

[16] Herbelot, O., (1992). Option Valuation of Flexible Investments: The Case of Environmental Investments in the Electric Power Industry, Ph. D., M.I.T., Massachusetts.

[17] Kinderlherer, D. And Stampacchia, G., (2000). An introduction to Variational Inequalities and its Applications, Second edition, SIAM, Philadelphia.

[18] Lions, J. L., (1969). Quelques méthodes de resolution des problémes aux limites non lineaires, Dunod, París.

[19] OKsendal, B., (1998). Stochastic Differential Equations. Springer $5^{\text {th }}$ edition, Berlin.

[20] PIndyck, R. S., (1986). Irreversible Investments, Capacity Choice and the Value of the Firm. American Economic Review, 78, 707-728.

[21] Scheinkman, J. A., (1994). Public goods and the Environment, in Environment Economics and their Mathematical Models, Díaz, J. I. and Lions, J. L. Eds, Masson, Paris, 149-158.

[22] Troianiello, G. M., (1987). Elliptic differential equations and obstacle problems. Plenum Press, New York.

\section{J. I. Díaz}

Dpto. de Matemática Aplicada

Facultad de Matemáticas

Universidad Complutense de Madrid

28040-Madrid, Spain

diaz.racefyn@insde.es

\section{Ch. Faghloumi}

Bayes Inference S. A.

Gran Vía, 39, $5^{\text {a }}$ planta

28013-Madrid,

Spain

cfaghloumi@bayesinf.com 